\title{
Digitalisierung der Medizin: Konsequenzen für die Ausbildung
}

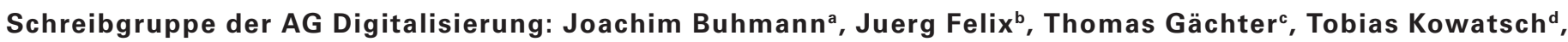
Roger Lehmanne, Nicola von Lutterotti ${ }^{f}$, Kuno Schedler ${ }^{9}$, Johann Steurer ${ }^{\text {, }}$, Christian Wolfrum ${ }^{i}$

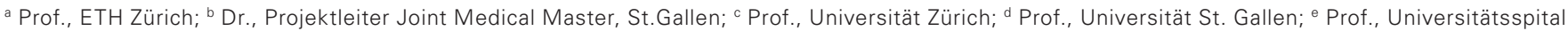

Zürich; ${ }^{\dagger}$ Dr., Wissenschaftsjournalistin, Zürich; ${ }^{g}$ Prof., Universität St. Gallen; ${ }^{h}$ Prof., Universität Zürich; ' Prof., ETH Zürich, für die Arbeitsgruppe "Digitalisierung der Medizin»

Die «Digitalisierung der Medizin» weckt grosse Hoffnungen auf eine effizientere und zunehmend bessere Medizin. Begriffe wie «Digitalisierung» und «künstliche Intelligenz» erzeugen zugleich aber auch Ängste. Dabei ist die Digitalisierung, die Erfassung und Speicherung von Daten in digitaler Form, nichts grundlegend Neues; neu hingegen sind die algorithmischen Fortschritte, kombiniert mit der enormen Leistungsfähigkeit moderner Computersysteme, aber auch die kostengünstige Speicherung und Übertragung grosser Datenmengen. Diese Errungenschaften haben das Potential, die Patientenversorgung merklich zu verbessern.

1 Der besseren Lesbarkeit halber wird die männliche Form verwendet.
Es ist davon auszugehen, dass die Entwicklungen im IT-Bereich die medizinische Profession grundlegend verändern - wie genau, lässt sich zwar noch nicht vorhersehen. Dennoch ist es an der Zeit, sich über die möglichen Entwicklungen und Herausforderungen Gedanken zu machen und zu planen, wie Mediziner ${ }^{1}$ die Zukunft ihres Berufs mitgestalten können. Innerhalb des «Bildungsnetzwerks Medizin» (www.

\section{Résumé}

La numérisation croissante de la médecine et le développement fulgurant de I'intelligence artificielle (IA) et de la robotique transformeront radicalement le métier de médecin. Un groupe de tr avail de «Bildungsnetzwerk Medizin" (Réseau de formation Médecine) s'intéresse plus précisément à la forme que pourrait revêtir ce changement et à ses répercussions sur la formation des futures générations de médecins. Trois points nous semblent particulièrement pertinents dans ce cadre: (1) Le stockage électronique des données, par exemple dans le cas du dossier de patient, comporte un risque fondamental d'accès par des personnes non autorisées. Les étudiants doivent donc apprendre à protéger au mieux les données des patients. (2) Ils devraient également saisir les concepts mathématiques et statistiques fondamentaux utilisés pour mettre au point les algorithmes, afin d'être en mesure de comprendre les résultats générés par I'IA (généralement des indications de probabilités). (3) Enfin, ils devraient être capables d'interpréter correctement ces probabilités et de les communiquer de manière claire.

medunet.ch) hat sich daher eine Arbeitsgruppe gebildet, um zu folgenden Fragen Stellung zu nehmen: Welche potentiellen Konsequenzen hat die Digitalisierung auf die medizinischen Tätigkeiten und Was könnte dies für die Ausbildung von Medizinern bedeuten?

\section{Wie könnte sich die Digitalisierung auf die medizinischen Tätigkeiten auswirken?}

Deutliche Konsequenzen erwarten wir in folgenden Bereichen der Medizin:

- Administration: Daten über Patienten (biographische Angaben, Anamnese, Medikamente, Laborbefunde, usw.) müssen nicht wiederholt erhoben und gespeichert werden. Die Anamnese wird standardisiert erhoben und die Daten direkt in eine zentrale elektronische Datenbank eingegeben. Auch werden Überweisungs- und Entlassungsberichte zukünftig grösstenteils maschinell erstellt. Die Einführung des elektronischen Patientendossiers erübrigt den Versand von Berichten, da dazu Berechtigte auf die Patientendaten von überall her zugreifen können.

- Medizinisches Wissen: Informationen zu fast allen medizinischen Themen sind heute im Internet zumeist frei und einfach erhältlich. Patienten benötigen daher dringend Beratung, um diese Informationen korrekt einzuordnen und zu bewerten. 
- Interpretation von "medizinischen Bilddaten": Mit den neuen Technologien des «machine learning» gelingt es zunehmend besser, Röntgenbilder, MRI und CT-Aufnahmen und andere medizinische Bilddaten zu interpretieren.

- Digitale Biomarker: Mit elektronischen Tools wie Smartphone und Sensoren generierte digitale Biomarker erlauben die Quantifizierung von Parametern wie Gangqualität und Balance, aber auch soziale Interaktionen. Ihre Verwendung in Diagnostik und Therapie wird in Zukunft neue Behandlungsansätze ermöglichen.

- Einordnung und Vermittlung der KI-Ergebnisse: Im Computer oder im Internet verfügbare Algorithmen werden den Arzt bei diagnostischen und therapeutischen Entscheidungen unterstützen. Auch in Zukunft wird es primär Aufgabe des Arztes sein, die von Algorithmen generierten Ergebnisse zu interpretieren und dem Patienten, möglicherweise unterstützt von digitalen "Coaches», zu erläutern.
Diese Beratungstätigkeit wird an Komplexität, aber auch an Bedeutung zunehmen. Denn Algorithmen liefern grösstenteils Wahrscheinlichkeitsangaben, die nicht immer einfach zu verstehen sind. Ihre Relevanz dem Patienten zu erklären, ist eine äusserst anspruchsvolle Tätigkeit.

- Einbindung von Robotik und KI: Bei diagnostischen und therapeutischen "Interventionen» werden zunehmend Roboter oder auch «augmented», «mixed» oder "virtual reality» zum Einsatz kommen. Gewebeproben, um ein Beispiel zu nennen, können mit Unterstützung von Robotern oft schonender und präziser entnommen werden; und mit Hilfe von "augmented reality» lassen sich chirurgische Eingriffe, etwa das Platzieren von Schrauben in Wirbelsäulenfortsätzen, präziser und sicherer ausführen als mit manuellen Operationstechniken.

- Elektronische Kommunikation: Interaktionen zwischen Arzt und Patient dürften zunehmend elektronisch erfolgen. So können Patientendaten

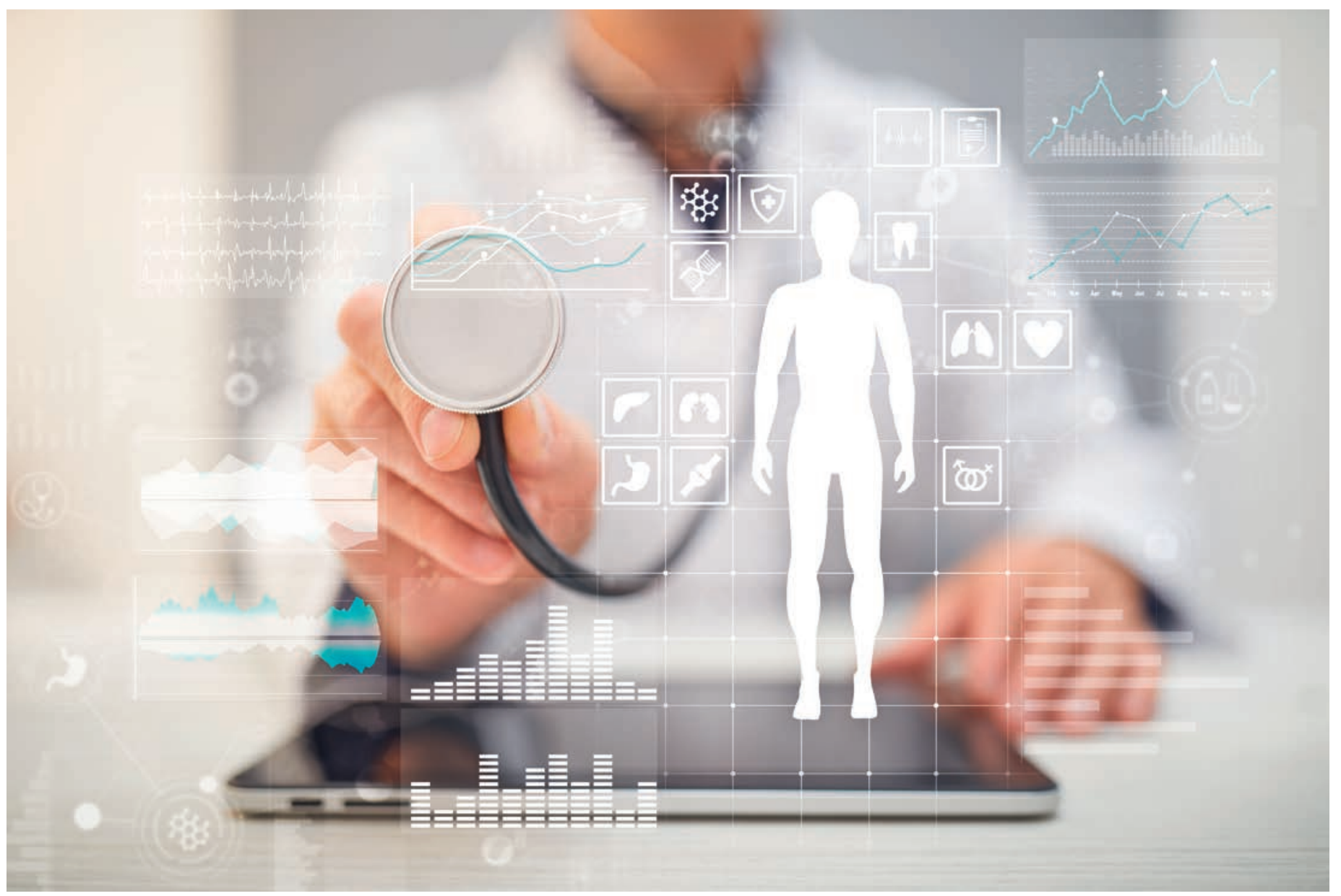

Die zunehmende Digitalisierung der Medizin und die rasante Entwicklung von künstlicher Intelligenz und Robotik werden die ärztliche Profession dramatisch verändern, so die Autoren. 
wie Hautveränderungen oder Blutzuckerwerte per Computer oder Smartphone an den Arzt übermittelt und von diesem auf dem gleichen Weg kommentiert werden. Auch hier ist denkbar, dass digitale Coaches, gleichsam als verlängerter Arm des Arztes, den Patienten therapeutisch begleiten und ihn bei der Datenerhebung für die nächste (telemedizinische) Sprechstunde unterstützen.

\section{Was bedeutet der digitale Wandel für die Ausbildung von Medizinern?}

Traditionell werden neue Inhalte in das Medizinstudium erst integriert, wenn sie als "gesichertes Wissen" gelten. Bei der «Digitalisierung» wäre dieses klassische Vorgehen verhängnisvoll. Denn hier kommt es darauf an, zukünftige Entwicklungen zu antizipieren, um diese mitgestalten zu können. Was auf uns zukommt, lässt sich zwar nicht genau vorhersehen, doch auf Basis der bisherigen Entwicklungen recht gut abschätzen. Um die medizinische Ausbildung an diese anzupassen, schlagen wir Folgendes vor:

1) Umgang mit Daten und Datensicherheit: Gesundheitsdaten sind besonders schützenswert. Die Digitalisierung erhöht die Gefahr, dass sich Unbefugte Zugang zu den Gesundheitsdaten verschaffen. Viele Gesetze (beispielsweise das Datenschutzgesetz, das Humanforschungsgesetz oder das Bundesgesetz über genetische Untersuchungen beim Menschen) sehen daher Vorschriften vor, um das Persönlichkeitsrecht der Patienten zu schützen. Ärzte müssen diese kennen und in ihrem Berufsalltag berücksichtigen.

Mit der Digitalisierung stellen sich zudem neue Herausforderungen, wie der «informed consent» ausgestaltet sein soll, um den rechtlichen Ansprüchen zu genügen. Worüber und wie umfassend muss beispielsweise der Patient aufgeklärt werden? Unter welchen Umständen kann eine digitale Aufklärung das klassische Arztgespräch ersetzen? Die wachsende technische Komplexität, etwa beim «machine learning» oder den robotergestützten Eingriffen, sowie die exponentielle Zunahme des medizinischen Fortschritts verändern zudem die Ausgangslage, was die ärztlichen Berufspflichten und die ärztliche Haftung betrifft. Von Interesse ist in diesem Zusammenhang etwa, ob, wie schnell und wie umfassend Ärzte neue Technologien in ihren Berufsalltag integrieren müssen und was dies für die Sorgfaltspflicht bedeutet. Auch stellt sich die Frage, wie bei einem Schadenseintritt die Verantwortung zwischen «Maschine» (bzw. Medizinproduktehersteller) und «Arzt» ausgestaltet sein soll.

\section{Lernziele:}

a) Die Studierenden sollen wissen, in welcher Weise Patientendaten geschützt sind und wie sie diesen Schutz in der Praxis gewährleisten können.

b) Es muss ihnen bewusst sein, auf welche digitalen Ressourcen sie für eine sach- und fachgerechte Behandlung zugreifen können und müssen.

c) Sie sollen in der Lage sein, dem Patienten das digital vorhandene Wissen so gut zu vermitteln, dass dieser sein Selbstbestimmungsrecht wahrnehmen kann.

2) Theorie algorithmischer Entscheidungsfindung in der Medizin: Algorithmen werden zunehmend in Diagnose, Prognose und Therapie eingesetzt. Dabei treten sehr anspruchsvolle informatische, statistische und kontrolltheoretische Probleme auf, die aus unserer Sicht auch den Algorithmenentwurf beeinflussen werden. Als Eingabe für Algorithmen in allen drei Aufgabenfeldern dienen Patientendaten, die meist mit Unsicherheiten behaftet sind. Entsprechend liefern die Algorithmen statistische Schätzungen, die validiert werden müssen. Die Korrektheit dieser Algorithmen muss im statistischen Kontext bewertet werden. Die Diagnose kann als Klassifikation eines Krankheitsbildes interpretiert werden, wobei statistische Einflüsse sowie fehlende Daten die statistische Schlussfolgerung (Inferenz) erschweren. Die Prognose kann als Vorhersage des Krankheitsverlaufs und damit als Regressionsproblem modelliert werden. Diese Vorhersagen müssen für algorithmische Entscheidungs- und Prognosesysteme validiert werden. Die Therapie wirft zudem schwierige konzeptionelle Fragen zur optimalen Kontrolltheorie auf. Dieser neue Aspekt erfordert Ergänzungen in der Ausbildung von Medizinern, um ihnen ein Grundverständnis von komplexen Systemen und der optimalen Kontrolle zu vermitteln.

Moderne Machine Learning-Systeme sind für den Benutzer wegen ihrer hohen Komplexität oft nicht mehr im Detail verständlich. Deshalb müssen zukünftige probabilistische Diagnose-, Prognose- und Therapieplanungssysteme mit automatisierten Validierungsmethoden ausgestattet sein, die algorithmische Ergebnisse bzgl. ihrer Validität einordnen können.

\section{Lernziele:}

a) Studierende können mathematische Konzepte auf medizinische Fragestellungen anwenden, wie z.B. die Erstellung von Prognosen, Modellierung von Therapien oder Tumorentwicklung. 
b) Sie haben ein Grundverständnis für die Gesetze des Zufalls und des Denkens in Wahrscheinlichkeiten; sie verstehen statistische Methoden bei medizinischen Fragestellungen und können sie anwenden.

c) Sie haben ein Grundverständnis für moderne Klassifikationsmethoden, insb. «random forests», «Deep Neural Networks».

d) Sie haben ein Grundverständnis für Bayesianische Inferenz, Bias Variance tradeoff

e) Sie haben ein Grundverständnis der Linearen Regression, von Gaussschen Prozessen und KernelMethoden.

f) Studierende haben Grundkenntnisse der Systemund Kontrolltheorie.

3) Interpretation und Kommunikation der Ergebnisse von Algorithmen: Die Resultate diagnostischer und prognostischer Algorithmen sind Wahrscheinlichkeiten und daher immer mit «Unsicherheit» verbunden. Für die Patienten, aber auch für die behandelnden Mediziner stellt sich die Frage, wie die wahrscheinlichkeitsbasierten Entscheidungen und Empfehlungen angemessen kommuniziert werden. Sowohl die korrekt visualisierten Unsicherheiten in den Antworten, als auch alternative Ergebnisse der Analyse spielen hier eine wichtige Rolle. Das Gleiche gilt für den Kommunikationskanal, d.h. welche Informationen dem $\mathrm{Pa}$ tienten wie vermittelt werden (Arzt oder digitaler Coach).

\section{Lernziele:}

a) Studierende kennen die unterschiedlichen Konzepte von Wahrscheinlichkeiten (frequentistisch und «degree of belief»).

b) Sie wissen, wie Wahrscheinlichkeiten numerisch und graphisch dargestellt werden können.

c) Sie wissen, wie Informationen Wahrscheinlichkeiten verändern und können die Folgen der veränderten Wahrscheinlichkeiten beurteilen.

d) Sie können den Patienten/Angehörigen/Mitgliedern anderer Professionen Wahrscheinlichkeiten in verständlicher Form erklären bzw. mit Hilfe von digitalen Coaches erklären lassen und diese Wahrscheinlichkeiten in den Entscheidungsprozess und Behandlungspfad integrieren.

Mit der einfachen Verfügbarkeit medizinischer Informationen (von der Anatomie über gesunden Lebensstil bis zu Dosierungen und Nebenwirkungen von Medika- menten) stellt sich für die Ausbildung der Mediziner die grundlegende Frage, wieviel und vor allem welches Wissen noch vermittelt werden muss. Ein beträchtlicher Teil des Medizinstudiums besteht derzeit darin, Fakten und medizinische Details wiedergeben zu können. Medizinische Informationen, etwa anatomische Details oder Dosierungen von Medikamenten, sind indes zunehmend schnell und einfach elektronisch abrufbar. Dass Mediziner auch in Zukunft über ein gewisses Detailwissen verfügen sollten, um nicht ständig in elektronischen Datenbanken danach suchen zu müssen, steht ausser Frage. Aber welches Detailwissen genau hängt von der später gewählten Fachdisziplin ab. Antworten auf diese für das Curriculum entscheidenden Fragen müssen in separaten Arbeitsgruppen oder Kommissionen erarbeitet werden.

Die Digitalisierung wird den Arzt nicht ersetzen, jedoch neue Anforderungen an den Berufsstand stellen. Hierzu zählt die Fähigkeit, die von Algorithmen generierten Ergebnisse einzuschätzen und dem Patienten in verständlicher Weise zu vermitteln. IT-Kenntnisse und kommunikative Kompetenzen dürften in der Medizin daher eine wachsende Rolle spielen. Auch müssen sich die Ärzte von morgen darauf einstellen, neue technologische Entwicklungen in ihren beruflichen Alltag zu integrieren. Aufgabe der Lehre ist es, sie für diese neuen Herausforderungen zu rüsten.

Bildnachweis

(C) Kirill Ivanov | Dreamstime.com
Prof. Dr. med. Johann Steurer Horten Zentrum für praxisorientierte Forschung und Wissenstransfer Universität Zürich Pestalozzistrasse 24 CH-8091 Zürich Tel. 0442553198 johann.steurer[at]usz.ch

\section{Arbeitsgruppe «Digitalisierung} in der Medizin»

Die Mitglieder des Bildungsnetzwerks Medizin bildeten eine Arbeitsgruppe zum Thema Auswirkungen der Digitalisierung in der Medizin auf die Ausbildung. In einer ersten Sitzung wurden Ideen zum Thema gesammelt. Eine "Schreibgruppe» verfasste bei einer zweitätigen Klausur dann einen ersten Entwurf. Dieser wurde in einer weiteren Sitzung mit der gesamten Arbeitsgruppe diskutiert und die Kommentare in das Manuskript integriert. Weitere Mitglieder der Arbeitsgruppe waren: Universität Zürich/ Universitätsspital Zürich, Prof. Matthias. Baumgartner, Prof. Matthias Guckenberger, Prof. Jürg Hodler, Prof. Roger. Lehmann, Dr. Christian Schirlo; Universität Luzern, Prof. Verena Briner; Universität Basel, Prof. Thorsten Schwede; ETH Zürich, Prof. Jörg Goldhahn, Prof. Gunnar Rätsch; Universität St.Gallen/Kantonsspital St.Gallen, Prof. Sandro Stöckli; Schweizerisches Institut für Weiter- und Fortbildung, Dr. Werner Bauer. 